\title{
Analog Electronic Circuits to Model Cooperativity in Hill Process
}

\author{
Maria Waqas $^{1 a}$, Muhammad Khurram ${ }^{1 b}$, S.M. Razaul Hasan ${ }^{2}$ \\ RECEIVED ON 14.01.2019, ACCEPTED ON 26.07.2019
}

\begin{abstract}
In the field of computational biology, electronic modeling of bio-cellular processes is in vogue for about a couple of decades. Fast, efficient and scalable electronic mimetics of recurrently found bio-chemical reactions are expected to provide better electronic circuit simulators that can also be used as bio-sensors or implantable biodevices at cellular levels. This paper presents some possible electronic circuit equivalents to model dynamics of one such bio-chemical reaction commonly involved in many bio-cellular processes, specifically pathways in living cells, known as the Hill process. The distinguishing feature of this process is cooperativity which has been modeled in silicon substrate using a pair of transistors, one transistor driving current in the other the same way ligand binding to one receptor site controls the binding affinity of the other receptor sites. Two possible circuits have been proposed and compared to electronically model cooperativity of a Hill reaction. The main idea is to exploit the natural analogies found between structures and processes of a bio-cell and electronic transistor mechanics, to efficiently model fundamental bio-chemical reactions found recurring in bio-processes. These circuits can then be combined and rearranged quickly to form larger, more complex bio-networks, thus mitigating the intricacies involved in modeling of such systems.
\end{abstract}

Keywords: Analog Electronic Circuit Modeling, Hill Process, Computational Biology, Cytomorphic Hardware, Cooperativity.

\section{INTRODUCTION}

$\mathrm{S}$ tudy of various life forms has always been a subject of colossal interest to the researchers. It has long been identified as a topic of biology, and biologists as well as bio-scientists have proficiently been able to gather enormous amount of data through observation and experimentation. Advances in biosciences have unleashed powerful approaches to determine various components and underlying biochemistry of living cells too. The biocellular machinery might appear simple in structure at a glance, but is enormously complex in its working and networking abilities [1]. The complexity and size of these bio-cellular networks have made their comprehension task arduous by theory and experimentation alone, while opening up new opportunities for mathematicians, computer scientists and even engineers [1-2], resulting in emergence of the interdisciplinary field called Computational Biology. Versatile modeling approaches have been employed in this domain, from mathematical and analytical models to computer-based simulations, to help understand these complicated and sophisticated bio-networks better [1,3-5].

Although mathematical and computational modeling of bio-cellular entities and processes have interested researchers since many decades, electronic modeling of bio-cellular processes is an emerging research trend

${ }^{1}$ Department of Computer and Information Systems Engineering, NED University of Engineering and Technology, Karachi, Sindh, Pakistan. Email: ${ }^{a}$ mariaw@ @ neduet.edu.pk (Corresponding Author), ${ }^{\mathrm{b}}$ mkhurrum@ @eduet.edu.pk

${ }^{2}$ Centre for Research in Analog and VLSI Microsystems Design (CRAVE), Massey University, Auckland, New Zealand. Email: hasanmic@ massey.ac.nz

This is an open access article published by Mehran University of Engineering and Technology, Jamshoro under CC BY 4.0 International License. 
in biosciences as well as engineering. Computational models suffer from a potential requirement of big, power hungry machines to run simulations. Simulation of one whole-cell cycle of the smallest bacteria on earth, using an over simplified model, was run on a 128-node Linux cluster in 10 hours [6]. To overcome these issues an alternate direction in vogue nowadays is modeling of bio-cellular processes functionalities in specialized analog [2, 7-8], digital [9-11] or mixed IC electronic hardware [12-13]. The research work presented in this paper advocates analog transistor mimetics for bio-cellular processes as the bio-cellular processes and circuits are naturally more analog than digital; they are noisy and act in progressive way rather than stern off/on digital states [14-15]. Many logical similarities are found among parameters and processes of biological and electronic domains. Biological processes largely comprise of feedback processes and oscillators which are also common characteristics of many analog electronic domain devices. Digital electronic circuits employing merely two states, are unable to make use of complete informational capacity of an electronic transistor; which otherwise can work on a very wide range of values [16]. Thus transistor provides more versatile functionality when used in analog domain, fitting to biological circuits much more appropriately.

Although the bio-cellular processes are immensely complex in their interactions, a finite set of biochemical reaction underlie them. A modular approach is to efficiently model these fundamental bio-chemical reactions in analog electronic domain anticipating that this would make modeling of larger networks easy $[2,17]$. This paper presents an extension to the work published by the author in [17] in which two such processes namely receptor-ligand binding and Michaelis Menten reaction, were modeled using analog transistors in electronic domain. This work presents several approaches to model a third fundamental reaction called the Hill process with argumentative and simulation support. The same process has also been worked upon for equivalent electronic model in [2] but there are some key differences: One key difference is that [2] and its preceding work used MOS transistors operating in sub-threshold region or BiCMOS technology for electronic modeling, whereas electronic modeling present

ed in this work uses MOS transistors in analog domain, operating in triode and saturation regions. The second main difference is the approach used to mimic the bio-cellular processes. In [2], the Hill coefficient is represented by amplifying the difference between the voltages representing the respective molecular concentrations, whereas here this constant is matched to the device parameter mechanics of a single MOS transistor, thus simplifying the overall model. A new set of analogies between the two domain has been developed, and the concept has been validated mathematically as well analytically through reasoning and simulations.

Some recent significant contributions in related domains include [18], in which a new solver called Arco for complex cytomorphic and neuromorphic computations has been presented, and most recently [19], which presents a digitally reconfigurable cytomorphic electronic chip to simulate a variety of bio-cellular reaction networks. Same research group has to its credit significant work on noise of such circuits too [20]. Expanding the research to implementation of biosensors, they have also devised a biosensing device for measurement and control of cellular molecules [21].

Mimicking the functionalities of bio-cellular networks in analog and mixed IC hardware can help in producing improved simulators for bio-cellular networks and processes [7-8] that can aid in better understanding of biological behaviors and drug testing. This kind of specialized hardware, due to size compactness and power efficiency, would also be easier to integrate with synthetic biological circuits $[2,9,12]$; thus can be used as bio-sensors, and as implantable bio-devices as well.

\section{DETERMINISTIC MATHEMATICAL MODEL OF HILL PROCESS}

In organic cells, signals are propagated from one part of the cell to other through cell signaling pathways. These pathways comprise of cascaded proteins which interact with each other through various bio-chemical reactions to amplify and transport signals from one 
point to another [22-23]. One such reaction, receptorligand binding occurs when a ligand protein binds to a receptor protein to form product, the receptor-ligand complex. This reaction involves receptor having only one site where a ligand molecule can bind. An alternate phenomenon is cooperative binding, where the receptor has more than one identical binding sites. The receptor reacting in cooperative binding molecule is a multi-meric (multi-site) protein, where binding of one molecule of ligand to a receptor site affects the affinity of other ligand molecules towards the remaining receptor sites, resulting in non-linear cooperative behavior. Hence for positive cooperativity, a small increase in concentration of ligand molecules rapidly increases reaction rate. This kind of cooperative binding is exhibited when oxygen binds to Hemoglobin protein in blood. Hemoglobin transports oxygen through blood and this cooperative binding behavior makes this oxygen shuttling all the way efficient [2]. Hill function quantifies this cooperative binding and is modeled by the reaction given in Equation (1). In this reaction a receptor $R$ having $n$ binding sites, binds to $n$ ligand molecules, $L$ representing each ligand molecule, forming a receptorligand complex $R L_{n} . k_{1}$ and $k_{-1}$ respectively are the forward and reverse reaction rate constants.

$\mathrm{R}+\mathrm{nL} \underset{k_{-1}}{\stackrel{k_{1}}{\rightleftharpoons}} \mathrm{RL}_{\mathrm{n}}$

The steady state behavior as reported in [22] is given by Equation (2).

$$
[\mathrm{RL}]=\frac{[\mathrm{R}][\mathrm{L}]^{\mathrm{n}}}{\mathrm{K}_{\mathrm{d}}+[\mathrm{L}]^{\mathrm{n}}}
$$

where $[R],[L]$ and $[R L]$ represent the concentrations of receptor, ligand and receptor-ligand complex respectively. $K_{d}$ is called dissociation constant and is defined as $\mathrm{k}_{-1} / \mathrm{k}_{1} \cdot \mathrm{k}_{1}$ and $\mathrm{k}_{-1}$ have dimensions of concentration ${ }^{-1} \mathrm{sec}^{-1}$ and $\mathrm{sec}^{-1}$ respectively; while $\mathrm{K}_{\mathrm{d}}$ has units of concentration. [22] defines $\theta$ as the fraction of receptor sites bound at equilibrium, as shown in Equation (3).

$$
\theta=\frac{[\mathrm{L}]^{\mathrm{n}}}{\mathrm{K}_{\mathrm{d}}+[\mathrm{L}]^{\mathrm{n}}}
$$

Equation (3) produces graphs which are sigmoidal in shape. Here $\mathrm{n}$ is defined as the Hill's coefficient, and it regulates the steepness of the curve. From Equation (1) $n$ is expected to be the number of binding sites on the multimeric receptor, but experimental results show that this is the degree of interaction between the sites instead and never reaches the actual number of binding sites [22]. The more the cooperativity, the higher the value of $n$; hence steeper the curve. Monomeric receptor-ligand binding is a special case of Hill reaction with $n=1$, showing no cooperativity. Fig. 1 depicts the graphical results as discussed. The results clearly suggest that increasing $n$ makes the sigmoidal curve steeper and more switch-like. When value of the Hill coefficient is 1 , the plot becomes hyperbolic.

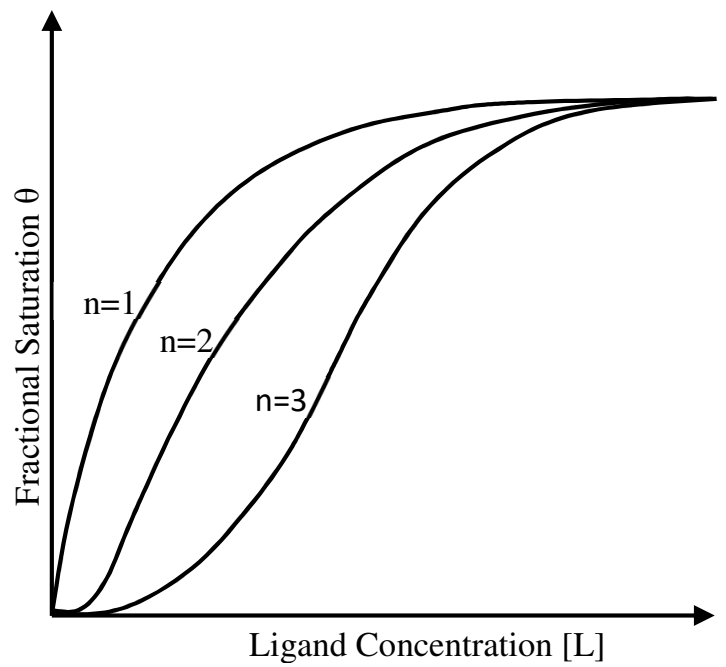

Fig.1: Hill Function Sigmoidal Curves. Fractional saturation $\theta$ plotted against Ligand Concentration [L] for different values of $n[19]$

\section{PROPOSED ANALOG ELECTRONIC MODELS FOR HILL PROCESS}

This section proposes some possible MOS transistor equivalents to model Hill process. The equivalences and electronic circuit validations stated in [17] suggest that a single MOS transistor operating in analog mode can model a non-linear bio-cellular reaction like receptor-ligand binding or Michaelis Menten kinetics. The drain and gate voltages are analogous to ligand and receptor concentrations respectively. For Hill process model, another transistor has been cascaded, whose gate voltage is proportional to the drain current of the first transistor as shown in Fig. 2, where these 
first and second transistors are labeled as $\mathrm{M}_{1}$ and $\mathrm{M}_{2}$. The gates of these transistors represent the two binding sites of a single bi-meric receptor. The proposed idea is that switching on of $\mathrm{M}_{2}$ is controlled by the drain current produced in $M_{1}$. Making $M_{2}$ reach saturation at a quicker rate as compared to the first one, it can be said that this second site provides faster binding rate if first site is already occupied; hence this transistor cascade can model increase in binding affinity of a site as a result of binding occurring at some other site of the same receptor molecule. The diodes D prevent the gate voltage of $\mathrm{M}_{2}$ from exceeding a prescribed gate voltage. $V_{[R]}$ and $V_{[L]}$ are the voltages respectively representing bi-meric receptor and ligand molecular concentrations, while $\mathrm{I}_{\mathrm{ds} 1}$ and $\mathrm{I}_{\mathrm{ds} 2}$ are the drain currents of transistors $M_{1}$ and $M_{2}$ respectively, proportional to receptor-ligand complex concentrations after the first and second bindings. As discussed in [17], the drain current $\mathrm{I}_{\mathrm{ds} 1}$ flowing through the short channel transistor $\mathrm{M}_{1}$ is given as Equation (4).

$$
\mathrm{I}_{\mathrm{ds} 1} \propto \frac{\mathrm{V}_{[\mathrm{R}]} \mathrm{V}_{[\mathrm{L}]}}{\mathrm{E}_{\mathrm{sat}} \mathrm{L}+\mathrm{V}_{[\mathrm{L}]}}
$$

where $E_{\text {sat }}$ is the critical electrical field at velocity saturation point and $\mathrm{L}$ is the length of the transistor. The gate drive voltage of transistor $\mathrm{M}_{2}$ is proportional to $I_{\mathrm{ds} 1}$, hence $\mathrm{I}_{\mathrm{ds} 2}$ is limited by $\mathrm{I}_{\mathrm{ds} 1}$ and is given as Equation (5).

$$
\mathrm{I}_{\mathrm{ds} 2} \propto \frac{\mathrm{V}_{[\mathrm{R}]} \mathrm{V}_{[\mathrm{L}]}{ }^{2}}{\left(\mathrm{E}_{\mathrm{sat}} \mathrm{L}+\mathrm{V}_{[\mathrm{L}]}\right)^{2}}
$$

As can be seen from Equation (5), the cascaded arrangement of transistors $M_{1}$ and $M_{2}$ produces a current $\mathrm{I}_{\mathrm{ds} 2}$ as a higher order equation, converting hyperbolic curve of mono-meric receptor binding to sigmoidal curve for a bi-meric receptor binding. Equation (5) is comparable to Equation (2) for $\mathrm{n}=2$.

To represent variation in Hill coefficient $n$, two modeling approaches have been proposed. In the first approach the hill coefficient $\mathrm{n}$ is adjusted via resistor $R_{h}$ and the channel length $L_{2}$ of the transistor $M_{2}$. The basic idea here is to reduce resistance to transistor drain current which is analogous to bio-chemical product formation; thus modeling increased affinity. For $\mathrm{n}=1$, when there is only one binding site, the drain current through transistor $M_{1}$ shows the rate of final product formation. When there are more than one binding sites, the production rate is measured as drain current of transistor $\mathrm{M}_{2}$, adjusting values of $\mathrm{R}_{h}$ and $\mathrm{L}_{2}$ to model different values of $n$. In the second approach, $n$ is modeled as channel width of the transistors. The proposed idea is again to reduce the resistance to drain current by widening the path of current flow. Increase in transistor channel width models increasing n. Fig. 3 shows the suggested electronic model where transistors $\mathrm{M}_{2}, \mathrm{M}_{3}$ and $\mathrm{M}_{4}$ channel widths are in increasing order respectively. Hence drain currents of these transistors respectively represent the production rates for increasing values of $n$.

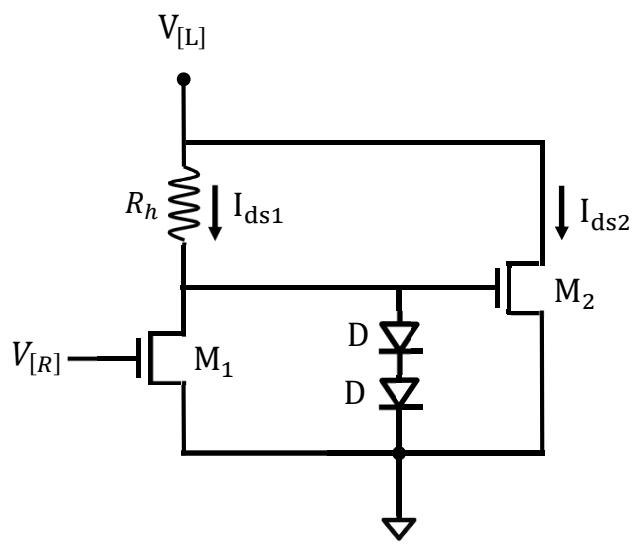

Fig. 2: Analog MOS Transistor Circuit Model for Hill Process, where $\mathrm{n}$ is represented by channel length of Transistor $\mathrm{M}_{2}$.

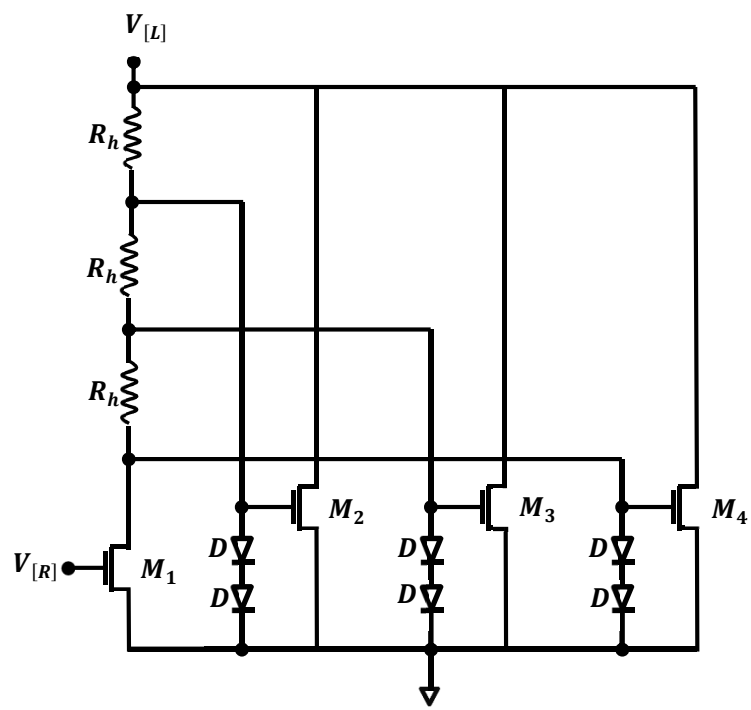

Fig. 3: Alternate Analog MOS

Transistor Circuit Model For Hill Process, where variation in is represented by adjusting channel width of Transistors 


\section{RESULTS AND DISCUSSION}

The circuits shown in Figs. 2-3 have been simulated in Cadence Virtuoso IC 5.1.41 and have been compared with Matlab simulations produced for Equation (2). The simulated curves of Matlab tally with the sigmoidal characteristic of Hill equation as reported in textbooks and other authentic literature of biochemistry.

Fig. 4 shows the graphical results for Hill process Equation (2) simulated in Matlab, for five different values of the Hill coefficient $n$. Receptor-ligand concentration RL is plotted against ligand ligand concentration $\mathrm{L}$ being varied from 0 to $5 \mathrm{mM}$, keeping $\mathrm{R}$ equal to $1 \mathrm{mM}, k_{1}$ to $1.2 \mathrm{KM}^{-1} \mathrm{sec}^{-1}$, and $\mathrm{k}_{-1}$ to 1 $\mathrm{sec}^{-1}$. These values have been adopted from data and results reported in [22-23]. As can be seen that when $\mathrm{n}$ is 1 the resulting curve is hyperbolic. When $\mathrm{n}$ is 2 the curve becomes sigmoidal showing the effect of cooperativity. As $\mathrm{n}$ increases further the curve becomes steeper or more switch like.

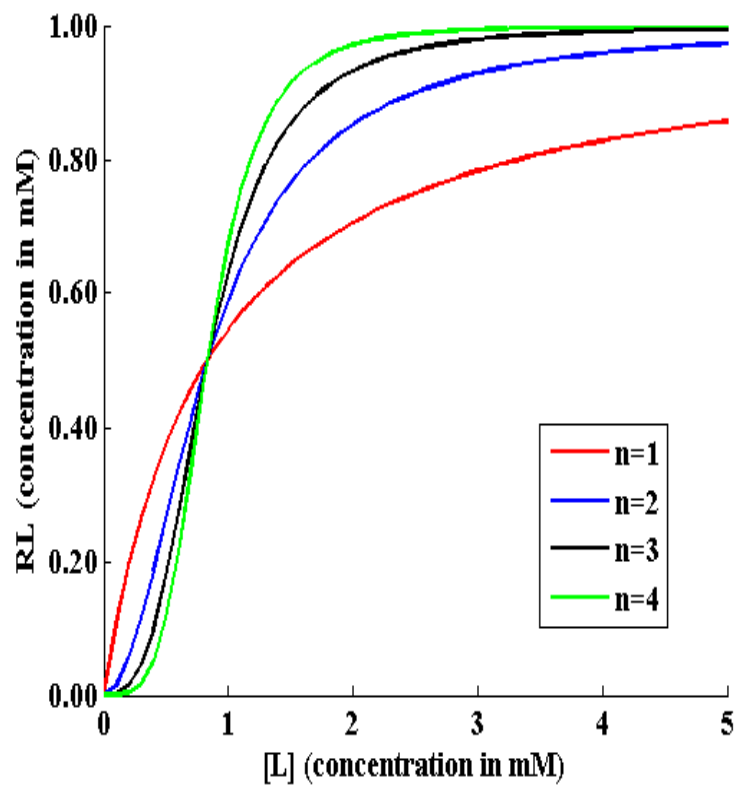

Fig. 4: Matlab Simulation results for Hill Equation as given in Eq. (2), illustrating the effect of changing Hill Coefficient $n$

Fig. 5 shows the graphical results after simulating the circuit of Fig. 2 in Cadence. For value of $n$ equal to 1 , voltage proportional to the drain current of $M_{1}$ is measured as $V_{[\mathrm{L}]}$ is varied from 0 to $3 \mathrm{~V}$. As stated earlier, this voltage represents the receptor-ligand complex concentration [RL] for a receptor having only one binding site. Here $V_{R}$ is kept $1.3 \mathrm{~V}, \mathrm{R}_{\mathrm{h}}$ is $2 \mathrm{~K} \Omega$, channel width for both $M_{1}$ and $M_{2}$ is $2 \mu \mathrm{m}$, and channel length for both the transistors is $750 \mathrm{~nm}$. Like Matlab simulation curves, it can be seen that when $\mathrm{n}$ is 1 , the resulting curve is hyperbolic showing zero cooperativity. For value of $n$ greater than $1,[R L]$ is proportional to the drain current of $\mathrm{M}_{2}$. It shows that with increasing $\mathrm{V}_{[\mathrm{L}]}$, there is a negligible increase in drain current of $M_{2}$ initially, which is turned into a rapid increase as the drain current in $\mathrm{M}_{1}$ grows, generating a sigmoidal curve.

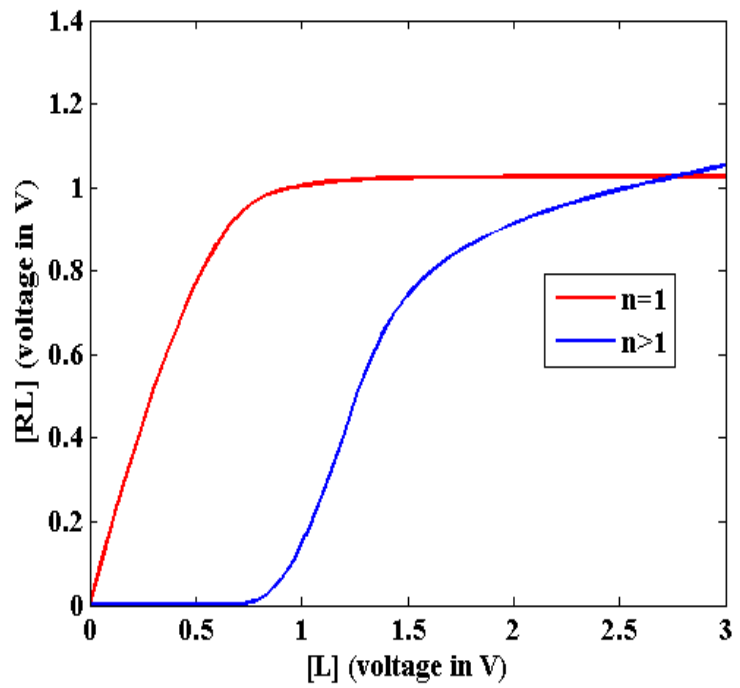

Fig.5: Graphical results for Hill Process obtained from cadence simulation of Electronic Circuit shown In Fig. 2, illustrating the effect of increasing binding sites on a receptor

When there are more than one binding sites, the production rate is measured at drain current of $\mathrm{M}_{2}$. For $\mathrm{n}$ being 2, 3 and 4, values taken for $\mathrm{R}_{\mathrm{h}}$ are $1 \mathrm{~K} \Omega, 3 \mathrm{~K} \Omega$ and $4 \mathrm{~K} \Omega$ respectively, and values for $L_{2}$ are $750 \mathrm{~nm}$, $550 \mathrm{~nm}$ and $350 \mathrm{~nm}$ respectively. The electronic simulation run in Cadence for this dataset produced graphical results as shown in Fig. 6. Graphical curves for four different values of $n$ follow the same trend as shown in Fig. 4 for Matlab simulation of Hill equation. For the second approach, where variation in $n$ is represented by changing transistor width, channel width of transistors $\mathrm{M}_{1}, \mathrm{M}_{2}, \mathrm{M}_{3}$ and $\mathrm{M}_{4}$ respectively are $2 \mu \mathrm{m}, 4 \mu \mathrm{m}, 6 \mu \mathrm{m}$ and $8 \mu \mathrm{m}$. Other parameters include $\mathrm{V}_{[\mathrm{R}]}$ to be $1.25 \mathrm{~V}, \mathrm{R}_{\mathrm{h}}$ to be $800 \Omega$, and channel length of all transistors to be $750 \mathrm{~nm}$. Like first approach here also $R_{h}$ adjusts the turning on time of the transistors. Graphical results obtained from 
Cadence simulation, when $\mathrm{V}_{[\mathrm{L}]}$ is varied from 0 to $5 \mathrm{~V}$, are shown in Fig. 7. These curves for [RL] vs [L] again show similar behavior as depicted in Fig. 4.

Simulation results for both the suggested electronic circuit equivalents for Hill process, as given in Figs. 67 , show similar progression as depicted in the Matlab graphs of Fig. 4.

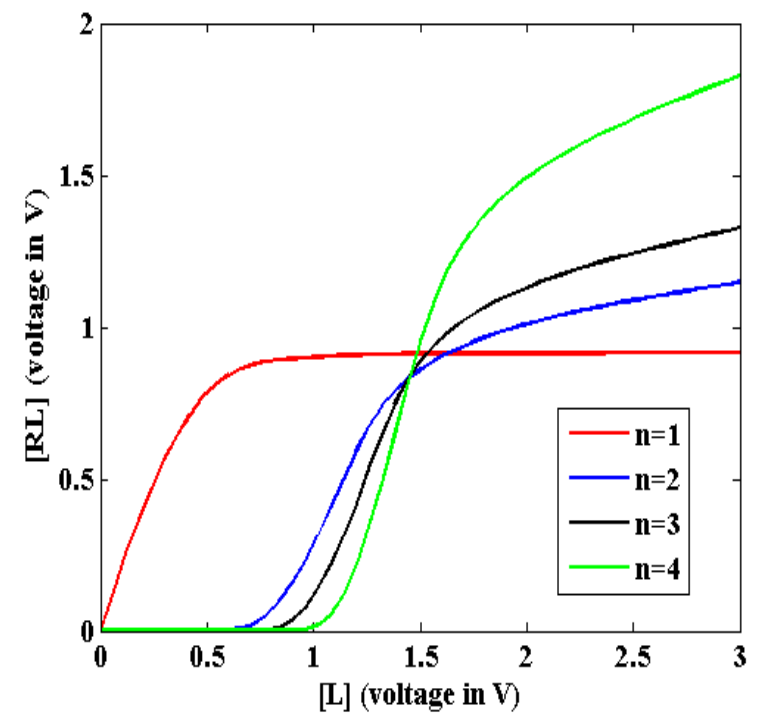

Fig. 6: Cadence simulation results for Hill Process obtained from The equivalent Electronic Circuit shown in Fig. 2, illustrating the effect of changing Hill Coefficient $n$

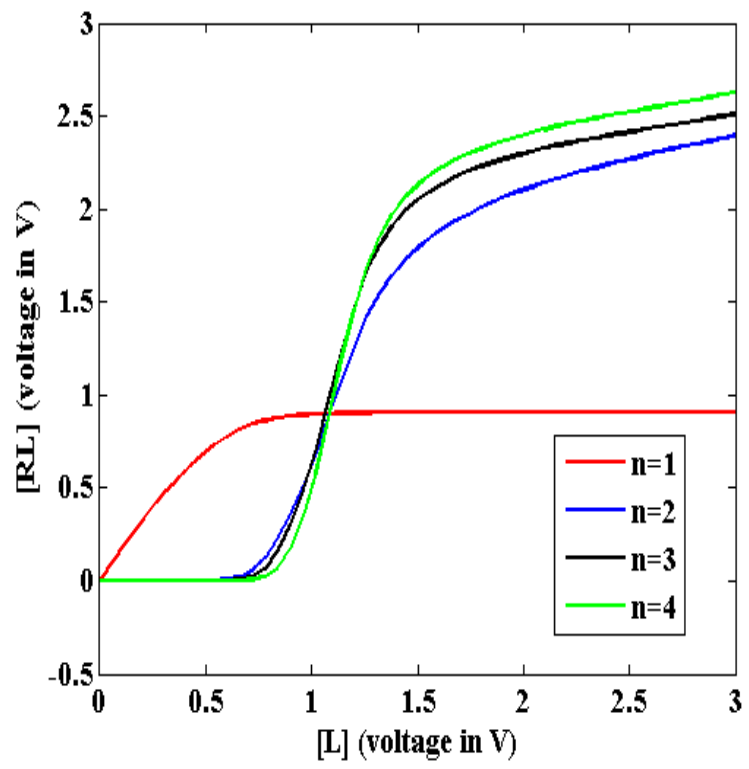

Fig. 7: Cadence simulation results for Hill Equation obtained from Electronic Circuit shown In Fig. 3, illustrating the effect of changing Hill Coefficient $n$.

\section{CONCLUSION}

This research endeavor has put forth some possible electronic models for the bio-cellular reaction called Hill process. To model dynamics of Hill process, two transistors have been used in cascade, one controlling the activation of the other. It has been shown that the drain current of the controlled transistor increases rapidly with increase in the drain current of the controlling transistor; the same way as ligand binding to one receptor site increases the ligand binding affinity of other site on the same receptor. These electronic circuits have been simulated in Cadence Virtuoso IC design software, producing the expected sigmoidal curves. To represent the Hill coefficient which governs the steepness of the sigmoidal curves, reduction in transistor drain current has been proposed which can be achieved by adjusting either length or width of the transistors. Results obtained for different circuit approaches have been compared quantitatively as well as qualitatively. The prospects of computational biology in electronic domain may seem promising in producing better, faster electronic simulators for bio-cellular processes; however, the work done so far in this domain is still in rudimentary stages. Researchers working in this area are trying different options; nevertheless, it is very early to comment on the qualitative order of these approaches. A lot of improvements can be applied to the circuits presented in this paper to gauge veracity of the results, still it puts forth some new dimensions to this research area.

\section{ACKNOWLEDGEMENTS}

Authors are thankful to the anonymous referees/experts for reviewing our work and giving valuable suggestions. Authors sincerely acknowledge the staff of High Performance Computing Center, NED University of Engineering \& Technology, Karachi, Pakistan, for providing easy access and technical support related to Cadence server.

\section{REFERENCES}

[1] Jong, H.D., "Modeling and Simulation of Genetic Regulatory Systems: A Literature Review", Journal 
Computational Biology, Vol. 9, No. 1, pp. 67103, January, 2002.

[2] Woo, S.S., Kim, J., and Sarpeshkar, R., “A Cytomorphic Chip for Quantitative Modeling of Fundamental Bio-Molecular Circuits", IEEE Transactions on Biomedical Circuits and Systems, Vol. 9, No. 4, pp. 527-542, August, 2015.

[3] Machado, D., Costa, R.S., Rocha, M., Ferreira, E.C., Tidor, B., and Rocha, I., "Modeling Formalisms in Systems Biology", AMB Express, Vol. 1, No. 1, pp. 45, December, 2011.

[4] Fisher, J., and Henzinger, T.A., "Executable Cell Biology", Nature Biotechnology, Vol. 25, No. 11, pp. 1239-1249, November, 2007.

[5] Taylor, A.S., Piterman, N., Ishtiaq, S., Fisher, J., Cook, B., Cockerton, C., Bourton, S., and Benque, D., "At the Interface of Biology and Computation", Proceedings of SIGCHI Conference on Human Factors in Computing Systems, pp. 493-502, New York, NY, USA, 2013.

[6] Karr, J.R., Sanghvi, J.C., Macklin, D.N., Gutschow, M.V., Jacobs, J.M., Bolival Jr, B., Assad-Garcia, N., Glass, J.I., and Covert, M.W., "A Whole-Cell Computational Model Predicts Phenotype from Genotype", Cell, Vol. 150, No. 2, pp. 389-401, July, 2012.

[7] Mandal, S., and Sarpeshkar, R., "LogDomain Circuit Models of Chemical Reactions", IEEE International Symposium on Circuits and Systems, pp. 2697-2700, 2009.

[8] Daniel, R., Woo, S.S., Turicchia, I., and Sarpeshkar, R., "Analog Transistor Models of Bacterial Genetic Circuits", IEEE Conference on Biomedical Circuits and Systems, pp. 333-336, 2011.

[9] Simpson, M.L., Cox, C.D., Peterson, G.D., and Sayler, G.S., "Engineering in the Biological Substrate: Information Processing in Genetic Circuits", Proceedings of IEEE, Vol. 92, No. 5, pp. 848-863, May 2004.

[10] Hasan, S.M.R., "A Micro-Sequenced CMOS Model for Cell Signaling Pathway using GProtein and Phosphorylation Cascade", 5th International Conference on Mechatronics and Machine Vision in Practice M2VIP, pp. 57-62, 2008.

[11] Hasan, S.M.R., “A Digital CMOS Sequential Circuit Model for Bio-Cellular Adaptive Immune Response Pathway Using Phagolysosomic Digestion: A Digital Phagocytosis Engine", Journal of Biomedical Science Engineering, Vol. 3, No. 05, pp. 470475, 2010.

[12] Hasan, S.M.R., "A Novel Mixed-Signal Integrated Circuit Model for DNA-Protein Regulatory Genetic Circuits and Genetic State Machines", IEEE Transactions on Circuits System Regular Paper, Vol. 55, No. 5, pp. 1185-1196, June, 2008.

[13] Alam, S., and Hasan, S.M.R., "Integrated Circuit Modeling of Biocellular PostTranscription Gene Mechanisms Regulated by MicroRNA and Proteasome", IEEE Transactions on Circuits System Regular Paper, Vol. 60, No. 9, pp. 2298-2310, September, 2013.

[14] Sauro, H.M., and Kim, K., "Synthetic Biology: It's an Analog World", Nature, Volume 497, No. 7451, pp. 572, May, 2013.

[15] "Ultra Low Power Bioelectronics," Cambridge University Press. [Online]. Available:

http://www.cambridge.org/si/academic/subje cts/engineering/circuits-and-systems/ultralow-power-bioelectronics-fundamentalsbiomedical-applications-and-bio-inspiredsystems. [Accessed: 22 ${ }^{\text {nd }}$ January, 2016].

[16] "Analog computing returns I MIT CSAIL." [Online]. Available: http://www.csail.mit.edu/analog_computing _returns. [Accessed: 11 ${ }^{\text {th }}$ January, 2017].

[17] Waqas, M., Khurram, M., and Hasan, S.M.R., "Bio-Cellular Processes Modeling on Silicon Substrate: Receptor-Ligand Binding and Michaelis Menten Reaction", Analog Integral Circuits Signal Processing, Vol. 93, No. 2, pp. 329-340, November, 2017.

[18] Achour, S., Sarpeshkar, R., and Rinard, M.C., "Configuration Synthesis for Programmable Analog Devices with Arco", Proceedings of 37th ACM SIGPLAN 
Conference on Programming Language Design and Implementation, pp. 177-193, New York, NY, USA, 2016.

[19] Woo, S.S., Kim, J., and Sarpeshkar, R., “A Digitally Programmable Cytomorphic Chip for Simulation of Arbitrary Biochemical Reaction Networks", IEEE Transactions on Biomedical Circuits System, Vol. 12, No. 2, pp. 360-378, April, 2018.

[20] Kim, J., Woo, S.S., and Sarpeshkar, R., "Fast and Precise Emulation of Stochastic Biochemical Reaction Networks with Amplified Thermal Noise in Silicon Chips", IEEE Transactions on Biomedical Circuits and Systems, Vol. 12, No. 2, pp. 379-389, April, 2018.

[21] Banerjee, A., Weaver, I., Thorsen, T., and Sarpeshkar, R., "Bioelectronic Measurement and Feedback Control of Molecules in Living Cells", Scientific Report, Vol. 7, No. 1, pp. 12511, October, 2017.

[22] Boyle, J., "Lehninger Principles of Biochemistry (4th Edition): Nelson, D., and Cox, M., Biochemistry and Molecular Biology Education, Vol. 33, No. 1, pp. 74-75, January, 2005.

[23] Ingalls, B.P., "Mathematical Modeling in Systems Biology: An Introduction", MIT Press, 2013. 